\title{
Laparoscopic Extended Right Hepatectomy with Biliary Reconstruction for Hilar Cholangiocarcinoma
}

\author{
Shin Nakahira, MD \\ Department of Digestive Surgery, National Hospital Organization Kure Medical Center and Chugoku \\ Cancer Center, Kure, Japan. \\ Department of Digestive Surgery, Kansai Rosai Hospital, Amagasaki, Japan. \\ E-mail: snakahira-gi@umin.ac.jp
}

Yutaka Takeda, MD

Department of Digestive Surgery, Kansai Rosai Hospital, Amagasaki, Japan.

Yoshiteru Katsura, MD

Department of Digestive Surgery, Kansai Rosai Hospital, Amagasaki, Japan.

Takeshi Kato, MD

Department of Digestive Surgery, Kansai Rosai Hospital, Amagasaki, Japan.

\section{Nobutaka Hatanaka, MD}

Department of Digestive Surgery, National Hospital Organization Kure Medical Center and Chugoku Cancer Center, Kure, Japan.

Shigeyuki Tamura, MD

Department of Digestive Surgery, Kansai Rosai Hospital, Amagasaki, Japan.

(C) Mary Ann Liebert, Inc. DOI: 10.1089/vor.2014.0247
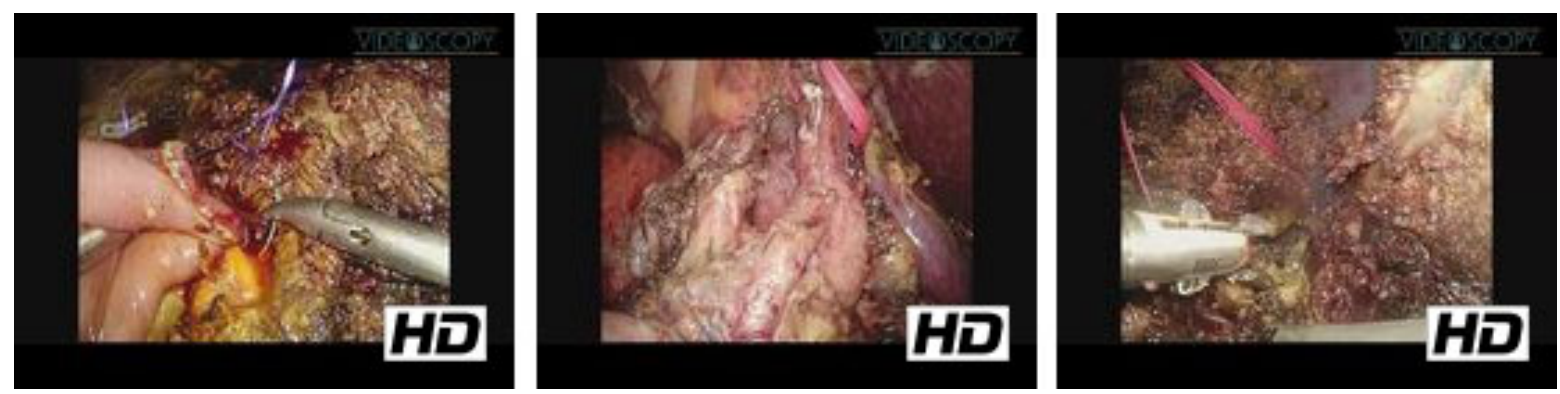

\section{Abstract}

Introduction: Although laparoscopic methods have been widely adopted for hepato-biliary-pancreatic surgeries, laparoscopic resection of hilar cholangiocarcinoma remains uncommon. ${ }^{1}$ One of the reasons is because it is a complicated procedure, consisting of major hepatectomy, lymph node dissection, and biliary reconstruction. There have been only one report about this procedure. ${ }^{1}$ From June 2010 through May 2013, 167 cases of laparoscopic hepatectomy (19 cases of lobectomy), 20 cases of laparoscopic distal pancreatectomy, and 10 cases of laparoscopic pancreaticoduodenectomy were performed in our hospital. Laparoscopic surgery for hilar cholangiocarcinoma is possible through the combination of those procedures.

Methods: The patient was a 60-year-old man with jaundice. Magnetic resonance cholangiography showed stenosis of both hepatic ducts. After 22 days of biliary drainage, the patient underwent surgery. The patient was placed in the left hemilateral position and five laparoscopic trocars were positioned. First, the right lobe and the caudate lobe were mobilized away from the inferior vena cava. After performing the Kocher maneuver, lymphadenectomy of the hepatoduodenal ligament was initiated from the superior margin of the duodenum and pancreatic head. The lymph nodes were dissected along the vessels using 
laparoscopic coagulating shears, and the common bile duct, the right hepatic artery, and the right portal vein were divided. After the Arantius ligament was divided, the hanging tape was placed between the right and middle hepatic veins, along the Arantius ligament. The surface of the liver and the small vessels were divided using laparoscopic coagulating shears. Thereafter, a hepatic parenchymal transection was performed using the crash and clamping method, with bipolar coagulation forceps, which are also referred to as BiClamps. The right hepatic vein was divided using a linear stapler. The left hepatic duct was clamped distally using an endovascular clip and divided adjacent to the umbilical portion. The specimen was retrieved through a minimal epigastric incision. Finally, end-to-side endoscopic hepaticojejunostomy was performed with running 4-0 PDS sutures. Two intra-abdominal drains were placed and the operation was completed.

Results: The total operative time was 867 minutes and the total blood loss was $100 \mathrm{~g}$. The postoperative course was uneventful, and the patient was discharged on the 16th postoperative day. A histological examination of resected specimens indicated a curative state with clear margins. There was no tumor recurrence 10 months after the operation.

Conclusions: Laparoscopic resections for hilar cholangiocarcinomas are safe and feasible in selected patients and when performed by surgeons with expertise in hepatic surgery and minimally invasive techniques. Further studies are still needed to confirm the benefit of this approach over conventional surgery for hilar cholangiocarcinoma.

No competing financial interests exist.

I have presented this work at the 2013 EAES congress in Vienna (Austria) on June 19-22, 2013.

Runtime of video: 5 mins 54 secs

\section{Cite this video}

Shin Nakahira, Yutaka Takeda, Yoshiteru Katsura, Takeshi Kato, Nobutaka Hatanaka, Shigeyuki Tamura, Laparoscopic Extended Right Hepatectomy with Biliary Reconstruction for Hilar Cholangiocarcinoma, J Laparoendosc Adv Surg Tech Part B Videoscopy. 2015, DOI: 10.1089/vor.2014.0247.

\section{References}

1. Gumbs AA, Jarufe N, Gayet B. Minimally invasive approaches to extrapancreatic cholangiocarcinoma. Surg Endosc 2013;27:406-414.

Original Publication Date: 2013 